\title{
Scientific Minimalism and the Division of Moral Labor in Regulating Dual-Use Research Steven Dykstra
}

\begin{abstract}
In this paper I examine the merits of a "division of moral labor" regulatory system for dual-use research. I borrow an argument from Thomas Douglas against scientific isolationism to show that researchers must be morally responsible for resolving at least some dual-use problems. I then argue that there are key benefits of scientific isolationism that are preserved in a position I call scientific minimalism. I then demonstrate that scientific minimalism, in a division of moral labor system, succeeds in maximizing both scientific freedom and moral efficiency, which I hold to be an essential aim for any proposed alternative regulatory model.
\end{abstract}

\section{Introduction and Clarifications}

The term "dual-use research" has evolved from its first conception to its present-day use. Originally defined as research that could be used for both military and civilian interests, it now refers to any research that can be used for both beneficial and harmful purposes. ${ }^{1}$ For the purposes of this paper I will borrow a clarification from Thomas Douglas, and refer to research wherein the potential negative consequences are sufficiently large, such that it is unclear whether or not the research should be pursued. ${ }^{2}$ This clarification allows the discussion to focus on the research that is most problematic and in need of investigation. Further, the phrase "dual-use problem" will refer specifically to a situation in which an agent is faced with the unclear decision of whether to pursue some form of dual-use research, rather than the debate about dual-use research in general.

The debate surrounding dual-use research is multi-faceted and has been accelerated in the last two decades by certain significant catalytic events. Researchers who took part in key discoveries regarding atomic fission were clearly involved in an early instance

\footnotetext{
${ }^{1}$ Michael Selgelid, "Dual-Use Research Codes of Conduct: Lessons from the Life Sciences," Nanoethics 3, no. 3 (2009): 175, doi:10.1007/s11569-009-0074-y.

2 Thomas Douglas, "The Dual-Use Problem, Scientific Isolationism, and the Division of Moral Labour,” Monash Bioethics Review 32 (2014): 86, doi: 10.1007/s40592-014-0004-9.
} 
of dual-use research, being aware of both its beneficial uses in medicine and energy and its malevolent uses in creating dangerous atomic weapons. This case shows that dual-use research is not a new concept, it is not confined to the life sciences, and has extreme consequences.

Recent events and research cases have shifted the focus of dual-use research debates to the life sciences and greatly accelerated their significance. The anthrax attacks following the September 11 attacks in the U.S. greatly augmented fears of bioterrorism around the world. ${ }^{3}$ Additionally, in recent studies, scientists accidentally produced a strain of mousepox that could kill mice that had been vaccinated against it, manufactured a polio virus from scratch based off a publicly accessible genome of the virus, and created a synthesized replica of the $1918 \mathrm{flu}$ virus that had killed between 20 and 100 million people. ${ }^{4}$ These studies include clear instructions on how to replicate them and are accessible to the public, allowing malevolent agents to potentially follow their instructions to create biological weapons. A very recent controversy in the debate regards gain-of-function research on certain viruses, by which changes to biological agents may cause them to become better at infecting their hosts. Replication of these studies by malevolent actors, as well as the possibility of an accidental release of a deadly synthetic virus from a laboratory, could be devastating. Hence, a moratorium on such research was called on October 17, 2014 to give time for experts to assess the dangers of dual-use, gain-of-function research. ${ }^{5}$ Considerations such as these shed light on the need for research into effective and efficient ways of regulating dual-use research. Considering the precedent set by such a moratorium, as well as a recent growth of academic interest in regulation and governance of dual-use research, I take the need for at least some form of regulatory measures as a given in this paper.

\section{The Moral Obligation for Individual Researchers}

The first questions to answer in an ethical discussion of dualuse research are these: are there any moral responsibilities concerning the decision to pursue dual-use research and, if so, why should they

\footnotetext{
${ }^{3}$ Selgelid, "Dual-Use Research Codes of Conduct," 177.

${ }^{4}$ Ibid.

${ }^{5}$ Andy Kilianski, Jennifer B. Nuzzo, and Kayvon Modjarrad, "Gain-of-Function Research and the Relevance to Clinical Practice,” The Journal of Infectious Diseases (2015): 1-2, doi: 10.1093/infdis/jiv473.
} 
fall on the researcher, as opposed to on some external regulatory agency? The position that an agent is exempt from making moral considerations when choosing whether or not to pursue dual-use research is what Douglas calls "scientific isolationism." This position holds that the direction of scientific inquiry can be decided solely on scientific considerations, and without respect to moral considerations at all. He first considers the broader position of "full isolationism," in which all agents, including scientists, the government, and the public, are exempt from moral considerations when faced with a dual-use problem. The rejection of this position entails that there is at least some moral responsibility tied to the pursuit of dual-use research. He then considers the narrower yet stronger position of "restricted isolationism," in which the exemption of moral responsibility applies only to the individual researcher or group of researchers. The rejection of this position entails that, in at least some cases, the individual researcher or research group has moral responsibilities when pursuing dual-use research.

The presumption Douglas raises against scientific isolationism is that knowledge is a tool that can be used both beneficially and harmfully. When decisions are required about the production of other types of tools of this sort, such as weapons or computers, we can reasonably expect that, at least in some cases, decisions about whether to create and distribute the tool are accompanied by an assessment of the likely uses of the tool. This "use-assessment" of the uses that a tool is likely to have is an obligation that we expect from producers and distributors, at least in some cases, regardless of whether it is always necessary. The idea is that there is an obligation, prior to producing and distributing a tool that is susceptible to both good and bad uses, to determine that it will not likely be used in primarily bad ways. Douglas argues that scientific knowledge, as a tool with both good and bad uses, carries the same obligation in at least some cases. ${ }^{6}$

The full scientific isolationist, in attempting to overcome this presumption, may argue for the intrinsic value of scientific knowledge. According to Douglas, this argument rests on two claims. The first is that scientific knowledge has "noninstrumental value." It is valuable outside of any additional value that it creates; it is intrinsically valuable, regardless of any morally good or bad instrumental uses it may have. ${ }^{7}$ The second claim is that this intrinsic value is sufficient to determine whether dual-use research should

\footnotetext{
${ }^{6}$ Douglas, "The Dual-Use Problem," 93.
}

${ }^{7}$ Ibid., 94. 
be pursued. This position holds that a research question with likely harmful instrumental uses or applications could be pursued solely on the grounds that it is a scientifically interesting question. It also frees all agents from moral obligations when deciding whether to pursue dual-use research. However, Douglas points out that, while it is true that scientific knowledge has intrinsic value, it does not follow from this that it should be the sole criterion by which research questions are pursued. ${ }^{8}$ Considering that scientific knowledge has both intrinsic and instrumental value, one would assume that both ought to be taken into account when resolving a dual-use problem. In order for the argument of the full isolationist to hold, then, it must be shown that the intrinsic value of scientific knowledge outweighs its instrumental value. However, such a claim is likely to be implausible when considering some implications it would have. For example, institutions representing the instrumental use of scientific knowledge, such as education, the military, or health care, would likely be found to be receiving a disproportionate amount of resources and would have to operate only to the extent that they contribute to scientific progress. ${ }^{9}$ More extremely, it could imply that funding should be taken away from helping terminally ill patients and relocated into pursuing scientifically interesting research. This argument by the full scientific isolationist does not hold, therefore it can be said that there is at least some moral responsibility involved in resolving a dual-use problem.

Douglas goes on to consider a narrower version of scientific isolationism that he claims to be a stronger position than that of full isolationism. In restricted isolationism, only individual researchers and research groups are exempt from the obligation to consider likely morally good and bad uses of their research. ${ }^{10}$ This position differs from full isolationism in that it accepts that there is some moral responsibility in deciding whether to pursue dualuse research. Full isolationism denies this responsibility altogether, whereas restricted isolationism simply deflects the responsibility from the individual researcher or research group to some external agency. Douglas explores an argument by the restricted isolationist that appeals to a division of moral labor system. ${ }^{11}$ In this system, external agencies are given the moral responsibility of conducting use-assessements, and individual researchers are given the moral responsibility to freely pursue scientific goals within the system of

\footnotetext{
${ }^{8}$ Ibid.

${ }^{9}$ Ibid.

${ }^{10}$ Ibid., 97.

${ }^{11}$ Ibid., 99.
} 
external regulations set up by these agencies. The argument is that the most efficient scientific system for serving the public good is one that allows free pursuit of research questions without regard to the potential uses and misuses knowledge. Scientists can disregard potential misuses of knowledge in this system because there is an external agency ensuring that the system does indeed serve the common good. Additionally, Douglas points out that this system is morally efficient for three reasons. First, it ensures that two separate agents do not redundantly perform the same moral job. Second, it ensures that different moral responsibilities are assigned to agents who are best suited to do them. Third, it allows for agencies to direct their moral assessments and regulations at categories of research, such that regulations and use-assessments are not always required for individual research projects. ${ }^{12}$

While this system is morally efficient, it still cannot fully exempt the researcher from moral responsibility concerning dualuse research in all circumstances for two reasons. First, a problem arises regarding the successful implementation of this system. The reason for this is that this system represents an ideal in which it is likely that external agencies, such as the government, will not fully or properly perform their moral duties consistently. If this were to happen, the moral responsibility would have to fall on the researcher at least until reform is made. Second, a situation can be imagined in which the implications of a dual-use research question is likely to cause significant harm despite external agencies fully performing their moral responsibilities. Consider a research question regarding scientific knowledge about a newly discovered type of biological weapon. In this situation, the external regulatory agencies could not have anticipated this new mechanism and, while they work to find a way to regulate it effectively, there is a period of time during which the pursuit of this research would result in significant harm despite the existing regulatory measures. In this situation, the moral responsibility to not pursue this research until proper regulation is created and implemented would fall on the researcher. This is due to the researcher lacking confidence in the moral capacity of the system. When this situation arises, a researcher is no longer able to have confidence. Hence, even in an efficient and fully functioning division of moral labor system, designed to deflect moral responsibility away from the researcher, individual researchers or research groups cannot be fully exempt from moral responsibility when deciding whether or not to pursue some dual-use research.

${ }^{12}$ Ibid., 101. 


\section{The "Scientific Minimalist" in the Division of Moral Labor System}

Because Douglas aims to reject scientific isolationism rather than affirm an alternative position, his analysis, while compelling, lacks some considerations that I find useful. First, an inversion of his argument against giving assent to the intrinsic value of scientific knowledge serves to demonstrate the importance of this intrinsic value, especially with respect to increasing its instrumental value. Second, viewing the division of moral labor system from a position that accepts moral responsibility for dual-use problems, in at least some circumstances, presents the system as a potentially successful model of regulation. It may be problematic to implement this ideal system, but exploring it has value in that it sheds light on some considerations that should be made in any inquiry into alternative regulatory models.

Douglas considers the implications of giving assent to the intrinsic value of scientific knowledge, but he does not consider the alternative position, in which instrumental value outweighs intrinsic value. It is easy to see how the instrumental value of scientific knowledge owes much to research pursued primarily for its intrinsic value. Take radium, for example, which was discovered in the name of science before it was known to be medically useful, or penicillin, which was discovered by accident. These beneficial instrumental applications of scientific knowledge came from the curiosity of scientific inquiry, without regard to its instrumental uses. Such examples demonstrate that scientific knowledge would likely not be as instrumentally valuable if it were not for scientific questions being pursued primarily for their intrinsic value. Pursuing only instrumentally beneficial scientific knowledge, paradoxically, results in less instrumentally beneficial knowledge being produced. It is reasonable to conclude that both intrinsic and instrumental knowledge should be considered when resolving dual-use problems. A regulatory system then, also should take both into consideration without giving assent to either.

The previously mentioned division of moral labor system is problematic for the scientific isolationist because it does not fully exempt her from moral responsibility in resolving dual-use problems. However, this is not a problem for what I call the "scientific minimalist," who accepts that she may have moral responsibility in dual-use problems, but prefers for this to be as infrequent of an occurrence as possible. The less often the scientific minimalist is 
faced with a dual-use problem, the freer she is to pursue scientifically interesting questions, which are both intrinsically and potentially instrumentally valuable. Something interesting happens to the dualuse problem in the division of moral labor system that is beneficial to the scientific minimalist. Recall that a dual-use problem arises when the likely harmful consequences are significant such that it is unclear whether or not to pursue the research. By this definition, many research questions that would pose a dual-use problem outside of the division of moral labor system do not pose a dual-use problem within it. This is because the researcher can clearly pursue most potentially harmful research questions with the confidence that she is operating in a system that is regulated to serve the common good. This confidence is shaken only when it is likely that the research will cause harm under the current regulatory measures or when an external agency fails to perform its moral responsibility. Under this system, then, the scope of the term "dual-use problem" narrows considerably, allowing the researcher to pursue many scientifically interesting questions that would pose a dual-use problem outside of it. Additionally, the benefit of the moral efficiency of the system is retained. Hence it can be said of this system that it attempts to maximize both the intrinsic and the instrumental value of scientific knowledge without systematically prioritizing either.

\section{Conclusion}

The "division of moral labor" approach to dual-use problems is not without its flaws. For example, it is an ideal that would be difficult to realize. If external agencies consistently failed to perform their moral responsibility to regulate the system properly, it would break down. Both moral efficiency and scientific freedom would return to the state they were in outside of the system. Moral responsibility would largely be in the hands of researchers, who do not wish to have it because it impedes their ability to pursue scientifically interesting research. If external agencies failed only occasionally to perform their moral responsibilities, the success of the system would depend on how frequently they failed, and whether fewer failures could be achieved. Two key characteristics make the division of moral labor approach desirable. First, it always has some agency responsible for moral considerations, because the researchers accept that it must sometimes be their responsibility. This is beneficial because in any system, there is always the possibility that some research will likely have harmful consequences within the 
framework of the system that can only be resolved by the agency of the researcher. Further, it allows for the system to enhance the scientific work of researchers by mostly relieving them of moral responsibility and allows for researchers to enhance the moral work of the system, by taking moral responsibility if the system fails. Second, it recognizes the equality and interdependence of the instrumental and intrinsic values of scientific knowledge and seeks to maximize both of them. Seeking to maximize each value contributes to both the scientific efficiency and the moral efficiency that the division of moral labor system has. It is also important to recognize the interdependence of the two values of scientific knowledge because its intrinsic value often serves to advance its instrumental value, and the instrumental value is a vital part of modern society that serves to produce much good as well as prevent much harm. Lastly, the two values must have equality because prioritizing one over the other results in undesirable implications. 